\title{
Ask yeast how to burn your fats: Lessons learned from the metabolic adaptation to salt stress
}

\author{
Amparo Pascual-Ahuir ${ }^{1 *}$, Sara Manzanares-Estreder ${ }^{1,2}$, Alba Timón-Gómez ${ }^{1,2, \#}$, Markus \\ Proft $^{2 *}$ \\ ${ }^{1}$ Department of Biotechnology, Instituto de Biología Molecular y Celular de Plantas \\ IBMCP UPV-CSIC, Universitat Politècnica de València, 46022 Valencia, Spain. \\ ${ }^{2}$ Department of Molecular and Cellular Pathology and Therapy, Instituto de \\ Biomedicina de Valencia IBV-CSIC, 46010 Valencia, Spain. \\ \# Current address: Department of Neurology, University of Miami Miller School of \\ Medicine, Miami FL, USA. \\ *Correspondence to M. Proft (mproft@ibv.csic.es) or A. Pascual-Ahuir \\ (apascual@ibmcp.upv.es)
}

\begin{abstract}
Here we review and update the recent advances in the metabolic control during the adaptive response of budding yeast to hyperosmotic and salt stress, which is one of the best understood signaling events at the molecular level. This environmental stress can be easily applied and hence has been exploited in the past to generate an impressively detailed and comprehensive model of cellular adaptation. It is clear now that this stress modulates a great number of different physiological functions of the cell, which altogether contribute to cellular survival and adaptation. Primary defense mechanisms are the massive induction of stress tolerance genes in the nucleus, the activation of cation transport at the plasma membrane or the production and intracellular accumulation of osmolytes. At the same time and in a coordinated manner, the cell shuts down the expression of housekeeping genes, delays the progression of the cell cycle, inhibits genomic replication and modulates translation efficiency in order to optimize the response and to avoid cellular damage. To this fascinating interplay of cellular functions directly regulated by the stress we have to add yet another layer of control, which is physiologically relevant for stress tolerance. Salt stress induces an immediate metabolic readjustment, which includes the up-regulation of peroxisomal biomass and activity in a coordinated manner with the reinforcement of mitochondrial respiratory metabolism. Our recent findings are consistent with a model where salt stress triggers a metabolic shift from fermentation to respiration fueled by the enhanced peroxisomal oxidation of fatty acids. We discuss here the regulatory details of this stress-induced metabolic shift and its possible roles in the context of the previously known adaptive functions.
\end{abstract}

Keywords salt stress, Saccharomyces cerevisiae, high osmolarity glycerol pathway, peroxisome, mitochondria, integrated stress adaptation, metabolic switch. 
Budding yeast is an attractive and powerful model for the investigation of abiotic stress responses (Ho and Gasch 2015). Important environmental challenges include temperature, $\mathrm{pH}$, oxidative, xenobiotic and osmotic stresses. Hyperosmotic or salt stress has been an exceptionally fruitful line of abiotic stress investigation, and particular aspects of the cellular adaptation induced by it have been revealed with great molecular resolution (Saito and Posas 2012). The simple addition of cell permeable salts such as $\mathrm{NaCl}$ or less permeable osmotically active compounds such as sorbitol provoke a critical stress to the yeast cell mainly because of an immediate loss of water from the cell and the accumulation of potentially harmful cations in the cell interior (Hohmann et al. 2007; Hohmann 2015). In the absence of an efficient and timely response, salt stress will lead to cell death. As one can expect, the cellular response to this stress has many different layers (Figure 1) and despite the intensive investigation in this field over the past decades we anticipate to still see many novel insights leading to an integrative view of the yeast osmostress response.

The main signaling pathway operating upon osmotic stress has been discovered over 20 years ago with the identification of the stress activated MAP kinase Hog1, which represents the downstream effector of the High Osmolarity Glycerol (HOG) pathway (Brewster and Gustin 2014). Since then, it has been shown that the regulatory functions of activated Hog1 are surprisingly complex (de Nadal et al. 2002; Saito and Posas 2012). Thus the HOG pathway is the central, although not the exclusive signaling route, which orchestrates a cellular rescue program upon osmotic and salinity challenge. One prominent function of Hog1 takes place in the nucleus, where it coordinates a complex change in the global gene expression program of the cell (Martinez-Montanes et al. 2010; de Nadal and Posas 2015). Many stress-responsive genes, which are normally expressed at very low rates or even actively repressed, are transiently induced. Activated Hogl promotes this burst of stimulated gene expression in an integrative manner, as it directly participates in the early events of chromatin remodeling and PIC formation (Alepuz et al. 2001; Proft et al. 2001; Proft and Struhl 2002; Alepuz et al. 2003; de Nadal et al. 2003; De Nadal et al. 2004; Mas et al. 2009; Ruiz-Roig et al. 2012), in the transcriptional elongation process (Proft et al. 2006), in mRNA processing, stability and export (Molin et al. 2009; Romero-Santacreu et al. 2009; Regot et al. 2013), and in the process of translation (Bilsland-Marchesan et al. 2000; Teige et al. 2001; Warringer et al. 2010). In a global view, RNA polymerase complexes are transiently reallocated from housekeeping to stress-responsive gene promoters in the yeast genome (Cook and O'Shea 2012; Nadal-Ribelles et al. 2012). This allows the preferential expression of genes involved in stress adaptation, while the expression of genes involved in cell growth and proliferation is shut down at the same time. Surprisingly, this profound reprogramming of gene expression is not essential for the tolerance of acute osmotic stress, as has been shown in engineered yeast cells with a membrane-sequestered Hogl kinase unable to induce gene expression upon stress (Westfall et al. 2008). However, the reinforced expression of stress response genes might be beneficial for the long-term tolerance to osmotic stress (Berry and Gasch 2008; Mettetal et al. 2008) and indeed it has been shown that the transcriptional response gradually declines after repeated exposure to salt stress (Rienzo et al. 2015). In this respect it is important to note that the main osmolyte system of yeast, the biosynthesis of glycerol, is also placed under a strong transcriptional control. Intracellular glycerol accumulation is essential for yeast cells to adapt to hyperosmolarity and salt stress (Hohmann et al. 2007). Many layers of control have been identified in the past, the majority of them again depend on the HOG pathway. The expression of glycerol biosynthesis structural genes is heavily induced upon stress 
(Albertyn et al. 1994; Ansell et al. 1997). Additionally, a controlled accumulation of glycerol inside the cell is achieved by the regulation of the glycerol facilitator Fps1 and the glycerol uptake system Stl1 (Tamas et al. 1999; Ferreira et al. 2005). Glycolytic flux regulation during the use of glycolytic intermediates for osmolyte productions appears to be another adaptive function of the HOG pathway upon stress (Petelenz-Kurdziel et al. 2013). Thus, glycerol accumulation is the critical cell volume control system employed by yeast cells upon salinity challenge (Hohmann 2015). Moreover, an intracellular excess of $\mathrm{Na}^{+}$under these conditions might be reduced by the direct stimulation of cation transporters at the plasma and vacuolar membranes (Proft and Struhl 2004; Li et al. 2012). Of note, the ability to reinstall normal ionic strength and osmolarity in the cytosol is important for an efficient transcriptional response (Proft and Struhl 2004; Vanacloig-Pedros et al. 2015). Hence cellular osmolyte control and nuclear genomic reprogramming mutually control each other upon salt stress.

Other adaptive mechanisms have been identified whose function is to arrest the cell cycle in order to optimally dedicate the cells resources towards stress adaptation and to avoid damage upon salt stress. The Hog1 kinase, once activated by stress, is the molecule in charge of stopping cell cycle progression in different stages (Saito and Posas 2012). This rapid and transient cell cycle arrest is physiologically relevant for the adaptation to osmotic stress, since mutants with a defect in this process are stress sensitive (Escote et al. 2004; Clotet et al. 2006; Duch et al. 2013b). Hence, sophisticated mechanisms exist in yeast, which assure proper stress adaptation before entering again the cell cycle (Clotet and Posas 2007; Sole et al. 2015). Hog1 targets both components of the basal cell cycle machinery and the expression of cyclins. Related with this controlled cell cycle delay, osmotic stress via the Hog1 kinase shuts down genomic replication in order to avoid conflicts between competing DNA and RNA polymerases (Duch et al. 2013a). In this case, Hog1 targets the DNA replication complex in order to avoid genomic instability caused by simultaneously active transcription and replication upon stress (Duch et al. 2013b).

Our recent work has revealed yet another level of adaptation, which occurs in yeast cells upon salt challenge. In this case we focused at the metabolic balance between fermentation and respiration and found that salt stress induces an immediate shift towards respiration by the induction of mitochondrial biomass and specific mitochondrial components (Pastor et al. 2009). Now we have found that salt stress also activates peroxisomal biogenesis and the oxidation of fatty acids via $\beta$-oxidation (Manzanares-Estreder et al. 2017), which altogether suggests a model where a successful salt stress adaptation depends on a transient metabolic shift towards the oxidative catabolism of internal fatty acids at peroxisomes and mitochondria (Figure 2). The first indication of this stress-regulated metabolic shift came from the observation that many mutants in mitochondrial structural components were salt sensitive (Pastor et al. 2009). Moreover, the Hog1 and the nutrient regulated Snf1 protein kinases are involved in the activated expression of genes encoding mitochondrial functions upon salt shock. One function of mitochondrial reinforcement upon stress seemed to be the counteraction of intracellular reactive oxygen species (ROS), since mitochondria defective mutants over-accumulated ROS, which was reverted by exogenous addition of antioxidants (Pastor et al. 2009). Furthermore, a proteomic analysis of salt adapted mitochondria revealed the accumulation of antioxidant functions (Martinez-Pastor et al. 2010). In our recent study, we further explore the implication of peroxisomes in yeast salt tolerance. Peroxisomal activity is important for the successful adaptation to high salinity, which becomes even more evident upon sugar limitation. The HOG pathway controls the transcriptional up-regulation of numerous genes involved in the activation 
of fatty acids from internal lipid stores, their internalization and $\beta$-oxidation in peroxisomes and their conversion into acetyl-carnitine (Manzanares-Estreder et al. 2017). The transcription factor in charge of this switch is Adr1, which was previously known to be regulated during the transition from fermentation to respiration via the Snf1 kinase (Ratnakumar and Young 2010). As a consequence, salt stress triggers the rapid induction of the cells $\beta$-oxidation activity and a failure in this process makes cells more vulnerable to this stress condition. Peroxisomal adaptation to salt stress is also microscopically evident, since the number of the organelle quickly increases upon exposure to high salinity. The dynamin related GTPases Dnm1 and Vps1 are involved in this stress-stimulated process, which depends on retrograde signaling but not the HOG pathway (Manzanares-Estreder et al. 2017). Overall, a yeast cell which actively combats a salinity insult, roughly doubles the number of peroxisomes attached to the mitochondrial network, and peroxisomal activity is actually needed to induce mitochondrial respiration under these conditions. This metabolic effort, which can be described as a transient diet change from sugar fermentation to the oxidative respiration of internal fats, is needed for salt adaptation. A very recent unbiased fitness survey using the yeast knockout collection under continuous osmotic stress indeed identified peroxisomal (as well as mitochondrial) functions as one of the most enriched functional category among the sensitive strains (Gonzalez et al. 2016). This underscores the physiological importance of peroxisomal metabolism upon salt stress.

The potential reasons why a yeast cell is forced to switch from sugar fermentation to oxidative degradation of its internally stored fats during salt stress might actually be numerous and in any case are illustrative for the importance of metabolic regulation in order to efficiently combat abiotic stresses (Figure 3). It has been known for a long time that even moderate $\mathrm{NaCl}$ concentrations strongly interfere with the uptake of sugars in yeast cultures (Wei et al. 1982). Thus the transporter activity of sugar permeases might be directly inhibited under high salinity stress. This would explain that osmotic stress very strongly induces the expression of several sugar uptake systems located at the plasma membrane as a plausible compensatory mechanism (Posas et al. 2000; Rep et al. 2000). Furthermore the rapid induction of glycerol biosynthesis might lead to a decrease in the metabolic flux in the lower glycolytic pathway. This might cause a metabolic bottleneck for the cell despite the fact that during the transient growth arrest upon salt stress glycolytic intermediates are not used for biomass production (Petelenz-Kurdziel et al. 2013). The massive glycerol biosynthesis could additionally lower the glucose uptake capacity by yet another mechanism, the formation of the toxic byproduct methylglyoxal. This intermediate is known to accumulate in yeast cells upon osmostress and hence triggers the induction of detoxifying enzymes such as Glo1, Gre2 or Gre3 (Aguilera and Prieto 2001; Rep et al. 2001; Maeta et al. 2005). But despite the induced detoxification, methylglyoxal might still trigger inhibitory effects in the cell upon stress, and one of the toxicity mechanisms has been recently revealed as the inhibition of sugar permeases (Yoshida et al. 2012; Roy et al. 2016). The importance of a high glycolytic flux for osmoadaptation is further highlighted by studies using alternative carbon sources. Osmotolerance is lower upon growth on the less fermentable carbon source galactose accompanied by a reduced accumulation of glycerol (Vanacloig-Pedros et al. 2015). Moreover, in the absence of a fermentable carbon source, yeast cells do no longer accumulate glycerol, and the adjustment of osmotic balance is further delayed (Babazadeh et al. 2017).

Taken together, we hypothesize that high salinity causes a nutritional stress in yeast cells, which is counteracted by different metabolic adjustments. The stress induced expression of the Mpc3 mitochondrial pyruvate carrier might indeed reflect the fact that 
the intracellular levels of lower glycolytic intermediates decrease upon salt stress (Timon-Gomez et al. 2013). In this scenario, the switch from the low affinity Mpc $1 / 2$ to the high affinity Mpc1/3 pyruvate carrier might be necessary to sustain mitochondrial function upon salt stress (Bender et al. 2015). Stress-activated peroxisomal $\beta$-oxidation seems to be the alternative compensatory route upon high osmolarity, which fuels the mitochondrial respiration by additional import of Acetyl-CoA. Thus, salt stress and nutritional stress are tightly connected. Indeed, sugar depletion caused by salt stress might actually be the reason why the Snfl protein kinase is activated upon these environmental conditions (Hong and Carlson 2007). As a consequence Snfl has been found to participate in diverse regulatory steps upon salt stress, for example in the transcriptional activation of mitochondrial respiration components or ion transporters (Ye et al. 2006; Pastor et al. 2009). We have also to consider that salt stress could directly inhibit mitochondrial activity, which would induce compensatory mechanisms to alleviate the mitochondrial damage. Indeed, harsh conditions of osmotic stress induce programmed cell death via mitochondrial dysfunction in yeast (Silva et al. 2005). Additionally, activated Hog1 MAP kinase directly induces gene expression via the Rtg1/3 transcription factors (Ruiz-Roig et al. 2012), which respond to mitochondrial dysfunction within the retrograde pathway (Sekito et al. 2000). Interestingly, Hog1 is required, at least partially, for the induction of mitophagy, although at present it is unknown whether autophagic control mechanisms of the mitochondria are relevant for salt stress adaptation (Mao et al. 2011).

Taken together, salt and osmotic stress tolerance is intimately linked to metabolic readjustments, which have emerged in the past years and will probably be extended in the future. These insights might also be important to better evaluate our experiments done with a "simple" stimulus such as the addition of salt to a growing yeast culture, which causes profound metabolic changes in the cell. We anticipate that similar metabolic readjustments might also be important for the adaptation to other abiotic stresses beyond salt stress.

Acknowledgments The work of the authors was supported by grants from Ministerio de Economía y Competitividad (BFU2011-23326 and BFU2016-75792-R).

Aguilera J, Prieto JA (2001) The Saccharomyces cerevisiae aldose reductase is implied in the metabolism of methylglyoxal in response to stress conditions. Curr Genet 39:273-283

Albertyn J, Hohmann S, Thevelein JM, Prior BA (1994) GPD1, which encodes glycerol-3-phosphate dehydrogenase, is essential for growth under osmotic stress in Saccharomyces cerevisiae, and its expression is regulated by the highosmolarity glycerol response pathway. Mol Cell Biol 14:4135-4144

Alepuz PM, de Nadal E, Zapater M, Ammerer G, Posas F (2003) Osmostress-induced transcription by Hot1 depends on a Hog1-mediated recruitment of the RNA Pol II. EMBO J 22:2433-2442

Alepuz PM, Jovanovic A, Reiser V, Ammerer G (2001) Stress-induced map kinase Hog1 is part of transcription activation complexes. Mol Cell 7:767-777

Ansell R, Granath K, Hohmann S, Thevelein JM, Adler L (1997) The two isoenzymes for yeast NAD+-dependent glycerol 3-phosphate dehydrogenase encoded by GPD1 and GPD2 have distinct roles in osmoadaptation and redox regulation. EMBO J 16:2179-2187 
Babazadeh R, Lahtvee PJ, Adiels CB, Goksor M, Nielsen JB, Hohmann S (2017) The yeast osmostress response is carbon source dependent. Sci Rep 7:990

Bender T, Pena G, Martinou JC (2015) Regulation of mitochondrial pyruvate uptake by alternative pyruvate carrier complexes. EMBO J 34:911-924

Berry DB, Gasch AP (2008) Stress-activated genomic expression changes serve a preparative role for impending stress in yeast. Mol Biol Cell 19:4580-4587

Bilsland-Marchesan E, Arino J, Saito H, Sunnerhagen P, Posas F (2000) Rck2 kinase is a substrate for the osmotic stress-activated mitogen-activated protein kinase Hog1. Mol Cell Biol 20:3887-3895

Brewster JL, Gustin MC (2014) Hog1: 20 years of discovery and impact. Sci Signal 7:re7

Clotet J, Escote X, Adrover MA, Yaakov G, Gari E, Aldea M, de Nadal E, Posas F (2006) Phosphorylation of Hsl1 by Hog1 leads to a G2 arrest essential for cell survival at high osmolarity. EMBO J 25:2338-2346

Clotet J, Posas F (2007) Control of cell cycle in response to osmostress: lessons from yeast. Methods Enzymol 428:63-76

Cook KE, O'Shea EK (2012) Hog1 controls global reallocation of RNA Pol II upon osmotic shock in Saccharomyces cerevisiae. G3 (Bethesda) 2:1129-1136

de Nadal E, Alepuz PM, Posas F (2002) Dealing with osmostress through MAP kinase activation. EMBO Rep 3:735-740

de Nadal E, Casadome L, Posas F (2003) Targeting the MEF2-like transcription factor Smp1 by the stress-activated Hog1 mitogen-activated protein kinase. Mol Cell Biol 23:229-237

de Nadal E, Posas F (2015) Osmostress-induced gene expression--a model to understand how stress-activated protein kinases (SAPKs) regulate transcription. FEBS J 282:3275-3285

De Nadal E, Zapater M, Alepuz PM, Sumoy L, Mas G, Posas F (2004) The MAPK Hog1 recruits $\mathrm{Rpd} 3$ histone deacetylase to activate osmoresponsive genes. Nature 427:370-374

Duch A, de Nadal E, Posas F (2013a) Dealing with transcriptional outbursts during S phase to protect genomic integrity. J Mol Biol 425:4745-4755

Duch A, Felipe-Abrio I, Barroso S, Yaakov G, Garcia-Rubio M, Aguilera A, de Nadal E, Posas F (2013b) Coordinated control of replication and transcription by a SAPK protects genomic integrity. Nature 493:116-119

Escote X, Zapater M, Clotet J, Posas F (2004) Hog1 mediates cell-cycle arrest in G1 phase by the dual targeting of Sic1. Nat Cell Biol 6:997-1002

Ferreira C, van Voorst F, Martins A, Neves L, Oliveira R, Kielland-Brandt MC, Lucas C, Brandt A (2005) A member of the sugar transporter family, Stllp is the glycerol/H+ symporter in Saccharomyces cerevisiae. Mol Biol Cell 16:20682076

Gonzalez R, Morales P, Tronchoni J, Cordero-Bueso G, Vaudano E, Quiros M, Novo M, Torres-Perez R, Valero E (2016) New Genes Involved in Osmotic Stress Tolerance in Saccharomyces cerevisiae. Front Microbiol 7:1545

Ho YH, Gasch AP (2015) Exploiting the yeast stress-activated signaling network to inform on stress biology and disease signaling. Curr Genet 61:503-511

Hohmann S (2015) An integrated view on a eukaryotic osmoregulation system. Curr Genet 61:373-382

Hohmann S, Krantz M, Nordlander B (2007) Yeast osmoregulation. Methods Enzymol 428:29-45 
Hong SP, Carlson M (2007) Regulation of snfl protein kinase in response to environmental stress. J Biol Chem 282:16838-16845

Li SC, Diakov TT, Rizzo JM, Kane PM (2012) Vacuolar H+-ATPase works in parallel with the HOG pathway to adapt Saccharomyces cerevisiae cells to osmotic stress. Eukaryot Cell 11:282-291

Maeta K, Izawa S, Inoue Y (2005) Methylglyoxal, a metabolite derived from glycolysis, functions as a signal initiator of the high osmolarity glycerol-mitogen-activated protein kinase cascade and calcineurin/Crzl-mediated pathway in Saccharomyces cerevisiae. J Biol Chem 280:253-260

Manzanares-Estreder S, Espi-Bardisa J, Alarcon B, Pascual-Ahuir A, Proft M (2017) Multilayered control of peroxisomal activity upon salt stress in Saccharomyces cerevisiae. Mol Microbiol 104:851-868

Mao K, Wang K, Zhao M, Xu T, Klionsky DJ (2011) Two MAPK-signaling pathways are required for mitophagy in Saccharomyces cerevisiae. J Cell Biol 193:755767

Martinez-Montanes F, Pascual-Ahuir A, Proft M (2010) Toward a genomic view of the gene expression program regulated by osmostress in yeast. OMICS 14:619-627

Martinez-Pastor M, Proft M, Pascual-Ahuir A (2010) Adaptive changes of the yeast mitochondrial proteome in response to salt stress. OMICS 14:541-552

Mas G, de Nadal E, Dechant R, Rodriguez de la Concepcion ML, Logie C, JimenoGonzalez S, Chavez S, Ammerer G, Posas F (2009) Recruitment of a chromatin remodelling complex by the Hog1 MAP kinase to stress genes. EMBO J 28:326336

Mettetal JT, Muzzey D, Gomez-Uribe C, van Oudenaarden A (2008) The frequency dependence of osmo-adaptation in Saccharomyces cerevisiae. Science 319:482484

Molin C, Jauhiainen A, Warringer J, Nerman O, Sunnerhagen P (2009) mRNA stability changes precede changes in steady-state mRNA amounts during hyperosmotic stress. RNA 15:600-614

Nadal-Ribelles M, Conde N, Flores O, Gonzalez-Vallinas J, Eyras E, Orozco M, de Nadal E, Posas F (2012) Hog1 bypasses stress-mediated down-regulation of transcription by RNA polymerase II redistribution and chromatin remodeling. Genome Biol 13:R106

Pastor MM, Proft M, Pascual-Ahuir A (2009) Mitochondrial function is an inducible determinant of osmotic stress adaptation in yeast. J Biol Chem 284:30307-30317

Petelenz-Kurdziel E, Kuehn C, Nordlander B, Klein D, Hong KK, Jacobson T, Dahl P, Schaber J, Nielsen J, Hohmann S, Klipp E (2013) Quantitative analysis of glycerol accumulation, glycolysis and growth under hyper osmotic stress. PLoS Comput Biol 9:e1003084

Posas F, Chambers JR, Heyman JA, Hoeffler JP, de Nadal E, Arino J (2000) The transcriptional response of yeast to saline stress. J Biol Chem 275:17249-17255

Proft M, Mas G, de Nadal E, Vendrell A, Noriega N, Struhl K, Posas F (2006) The stress-activated Hog1 kinase is a selective transcriptional elongation factor for genes responding to osmotic stress. Mol Cell 23:241-250

Proft M, Pascual-Ahuir A, de Nadal E, Arino J, Serrano R, Posas F (2001) Regulation of the Sko1 transcriptional repressor by the Hog1 MAP kinase in response to osmotic stress. EMBO J 20:1123-1133

Proft M, Struhl K (2002) Hog1 kinase converts the Sko1-Cyc8-Tup1 repressor complex into an activator that recruits SAGA and SWI/SNF in response to osmotic stress. Mol Cell 9:1307-1317 
Proft M, Struhl K (2004) MAP kinase-mediated stress relief that precedes and regulates the timing of transcriptional induction. Cell 118:351-361

Ratnakumar S, Young ET (2010) Snf1 dependence of peroxisomal gene expression is mediated by Adr1. J Biol Chem 285:10703-10714

Regot S, de Nadal E, Rodriguez-Navarro S, Gonzalez-Novo A, Perez-Fernandez J, Gadal O, Seisenbacher G, Ammerer G, Posas F (2013) The Hogl stressactivated protein kinase targets nucleoporins to control mRNA export upon stress. J Biol Chem 288:17384-17398

Rep M, Krantz M, Thevelein JM, Hohmann S (2000) The transcriptional response of Saccharomyces cerevisiae to osmotic shock. Hot1p and Msn2p/Msn4p are required for the induction of subsets of high osmolarity glycerol pathwaydependent genes. J Biol Chem 275:8290-8300

Rep M, Proft M, Remize F, Tamas M, Serrano R, Thevelein JM, Hohmann S (2001) The Saccharomyces cerevisiae Skolp transcription factor mediates HOG pathway-dependent osmotic regulation of a set of genes encoding enzymes implicated in protection from oxidative damage. Mol Microbiol 40:1067-1083

Rienzo A, Poveda-Huertes D, Aydin S, Buchler NE, Pascual-Ahuir A, Proft M (2015) Different Mechanisms Confer Gradual Control and Memory at Nutrient- and Stress-Regulated Genes in Yeast. Mol Cell Biol 35:3669-3683

Romero-Santacreu L, Moreno J, Perez-Ortin JE, Alepuz P (2009) Specific and global regulation of mRNA stability during osmotic stress in Saccharomyces cerevisiae. RNA 15:1110-1120

Roy A, Hashmi S, Li Z, Dement AD, Cho KH, Kim JH (2016) The glucose metabolite methylglyoxal inhibits expression of the glucose transporter genes by inactivating the cell surface glucose sensors Rgt2 and Snf3 in yeast. Mol Biol Cell 27:862-871

Ruiz-Roig C, Noriega N, Duch A, Posas F, de Nadal E (2012) The Hog1 SAPK controls the Rtg1/Rtg3 transcriptional complex activity by multiple regulatory mechanisms. Mol Biol Cell 23:4286-4296

Saito H, Posas F (2012) Response to hyperosmotic stress. Genetics 192:289-318

Sekito T, Thornton J, Butow RA (2000) Mitochondria-to-nuclear signaling is regulated by the subcellular localization of the transcription factors Rtg1p and Rtg3p. Mol Biol Cell 11:2103-2115

Silva RD, Sotoca R, Johansson B, Ludovico P, Sansonetty F, Silva MT, Peinado JM, Corte-Real M (2005) Hyperosmotic stress induces metacaspase- and mitochondria-dependent apoptosis in Saccharomyces cerevisiae. Mol Microbiol 58:824-834

Sole C, Nadal-Ribelles M, de Nadal E, Posas F (2015) A novel role for lncRNAs in cell cycle control during stress adaptation. Curr Genet 61:299-308

Tamas MJ, Luyten K, Sutherland FC, Hernandez A, Albertyn J, Valadi H, Li H, Prior BA, Kilian SG, Ramos J, Gustafsson L, Thevelein JM, Hohmann S (1999) Fps1p controls the accumulation and release of the compatible solute glycerol in yeast osmoregulation. Mol Microbiol 31:1087-1104

Teige M, Scheikl E, Reiser V, Ruis H, Ammerer G (2001) Rck2, a member of the calmodulin-protein kinase family, links protein synthesis to high osmolarity MAP kinase signaling in budding yeast. Proc Natl Acad Sci U S A 98:56255630

Timon-Gomez A, Proft M, Pascual-Ahuir A (2013) Differential regulation of mitochondrial pyruvate carrier genes modulates respiratory capacity and stress tolerance in yeast. PLoS One 8:e79405 
Vanacloig-Pedros E, Bets-Plasencia C, Pascual-Ahuir A, Proft M (2015) Coordinated gene regulation in the initial phase of salt stress adaptation. J Biol Chem 290:10163-10175

Warringer J, Hult M, Regot S, Posas F, Sunnerhagen P (2010) The HOG pathway dictates the short-term translational response after hyperosmotic shock. Mol Biol Cell 21:3080-3092

Wei CJ, Tanner RD, Malaney GW (1982) Effect of sodium chloride on bakers' yeast growing in gelatin. Appl Environ Microbiol 43:757-763

Westfall PJ, Patterson JC, Chen RE, Thorner J (2008) Stress resistance and signal fidelity independent of nuclear MAPK function. Proc Natl Acad Sci U S A 105:12212-12217

Ye T, Garcia-Salcedo R, Ramos J, Hohmann S (2006) Gis4, a new component of the ion homeostasis system in the yeast Saccharomyces cerevisiae. Eukaryot Cell 5:1611-1621

Yoshida A, Wei D, Nomura W, Izawa S, Inoue Y (2012) Reduction of glucose uptake through inhibition of hexose transporters and enhancement of their endocytosis by methylglyoxal in Saccharomyces cerevisiae. J Biol Chem 287:701-711

\section{Figure legends}

Fig. 1 Summary of the main adaptive mechanisms in budding yeast upon salt and hyperosmotic stress. Global gene expression is transiently shifted from "housekeeping" genes towards the activated expression of "defense genes". The major osmolyte produced upon osmotic stress is glycerol. Stimulation of membrane cation transport is responsible for ion homeostasis during salt stress. During the adaptive response, cells arrest the cell cycle in a regulated manner and inhibit genomic replication to avoid instability caused by simultaneous transcription and replication. Mitochondrial activity is reinforced during the stress adaptation, and the stimulation of peroxisomal $\beta$ oxidation activity is required to induce mitochondrial respiration and to efficiently adapt to salt stress. FA = fatty acid.

Fig. 2 Overview of the signaling events leading to peroxisomal activation upon salt stress. Genes encoding enzymes for fatty acid activation and peroxisomal $\beta$-oxidation are induced via the Adr1 transcription factor in a Hog 1 dependent manner. Peroxisomal proliferation is stimulated upon salt stress depending on retrograde signaling components and the Vps1 and Dnm1 GTPases. Stimulated oxidation of fatty acids reinforces mitochondrial respiration through the import of Acetyl-CoA via the carnitine shuttle Crc1. The mechanisms of stimulated glycerol production via Hogl and the transcription factor Hot1 upon salt stress are included because of its possible interference with intracellular pyruvate levels, which might be compensated by the induction of the high affinity pyruvate carrier via the Sko1 transcription factor.

Fig. 3 Summary of the possible physiological connections between salt and metabolic stress. The model predicts that high salinity causes a decrease in the glycolytic flux by direct inhibition of sugar transport, the deviation of glycolytic intermediates during glycerol biosynthesis and inhibition by toxic byproducts of osmolyte production. Compensatory mechanisms are the induction of the high affinity mitochondrial pyruvate carrier $\mathrm{Mpc} 1 / 3$, the induction of peroxisomal fatty acid $\beta$-oxidation activity to fuel mitochondrial respiration via alternative carbon sources and the activation of retrograde signaling in response to mitochondrial damage. 
Figure 1

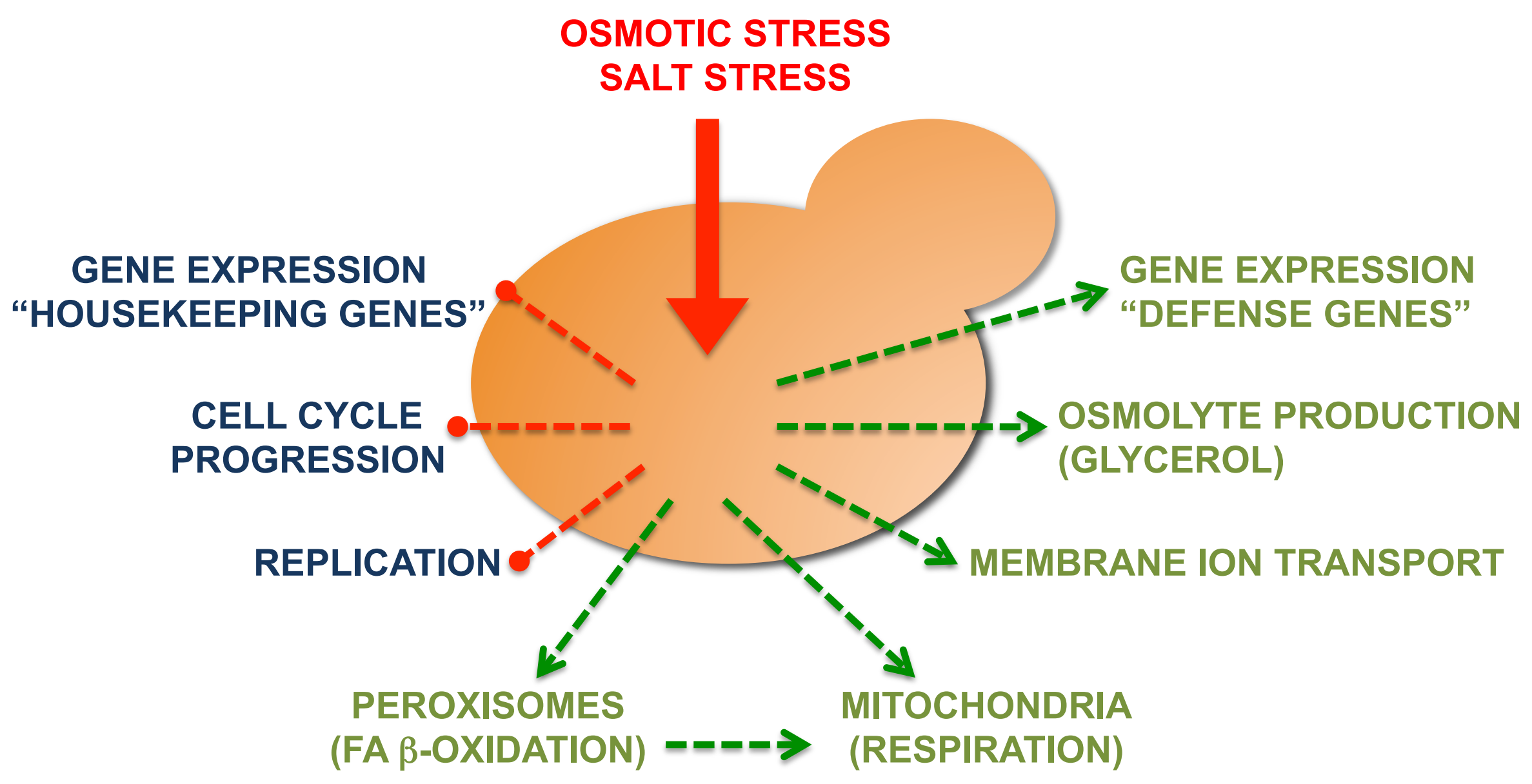




\section{Figure 2}

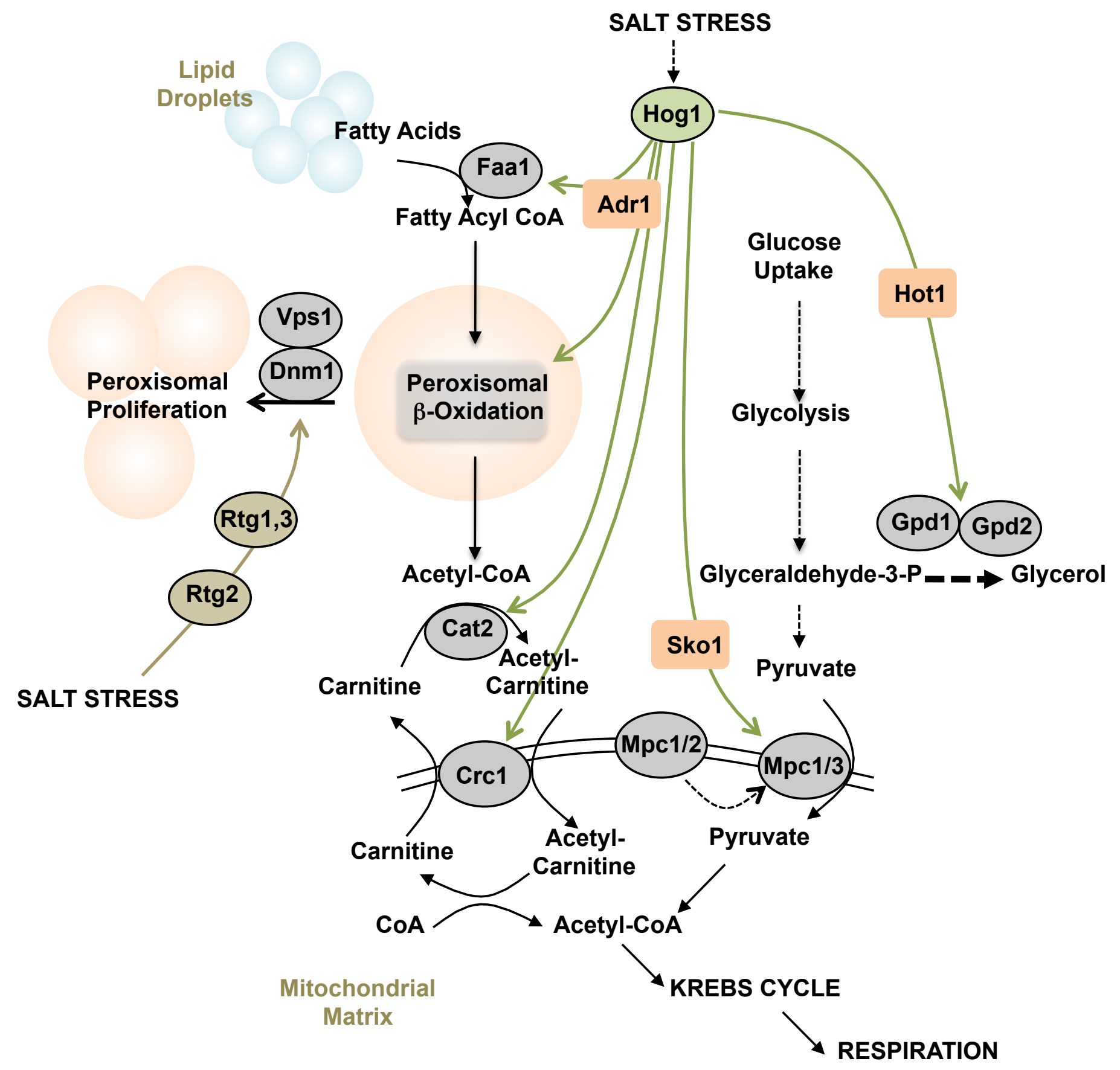


Figure 3

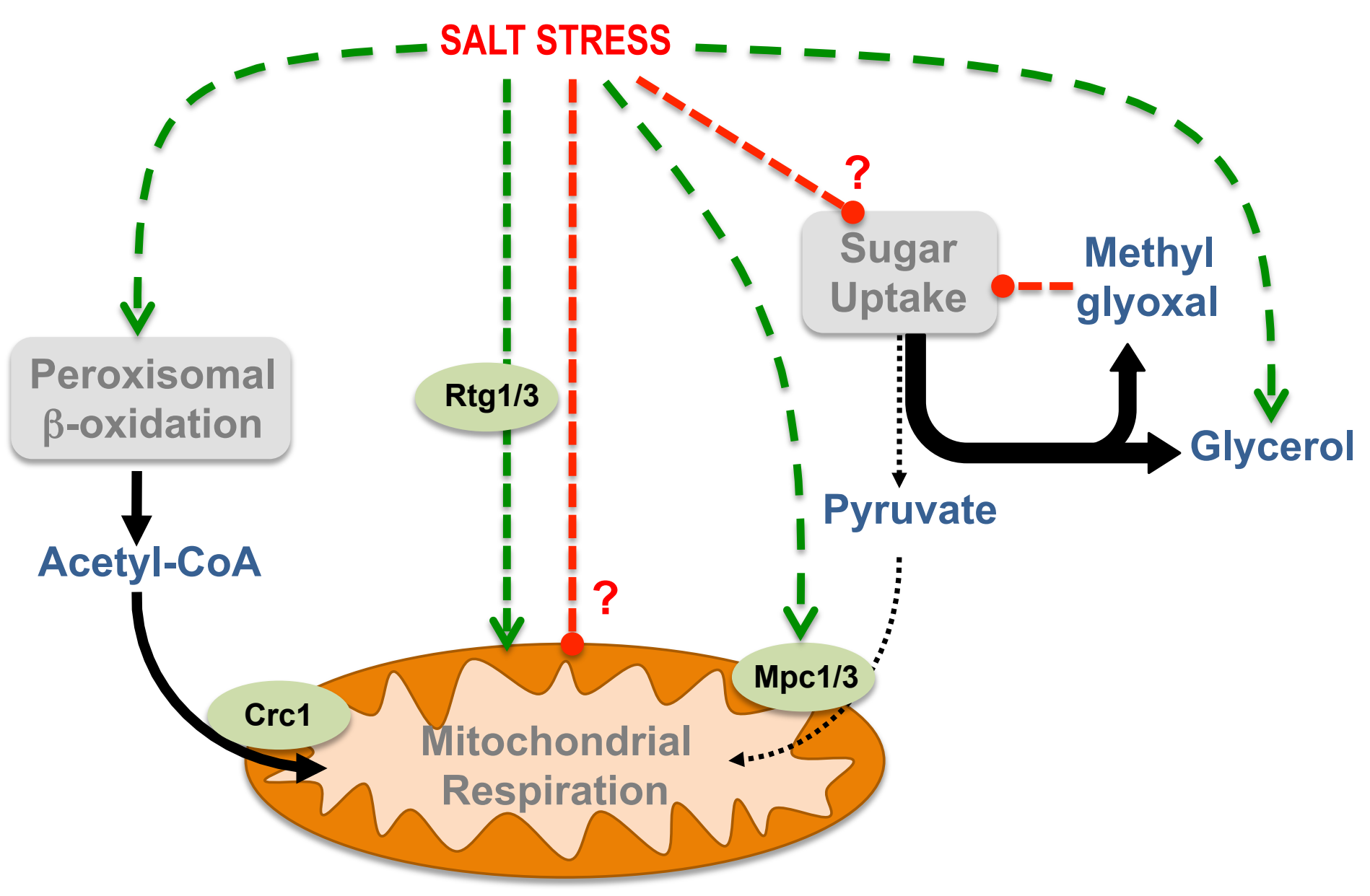

